\title{
Les fondations mythiques des cités de Lesbos: le témoignage d'Étienne de Byzance et de quelques autres...
}

Toute cité antique possède une date précise de fondation que, de nos jours, l'archéologie cherche à affiner au moyen de fouilles. C'est ce qu'on appelle le passé historique, étayé par toutes sortes de preuves matérielles et d'objets concrets. On peut alors donner à une cité en particulier une date plus ou moins précise au moyen de la chronologie absolue. ${ }^{1}$ Toutefois, rares sont les cités qui se contentent de ce passé historique, dûment prouvé et prouvable. Presque toutes font remonter leur origine beaucoup plus haut, dans un passé mythique, à l'époque très lointaine, où, croyait-on, les dieux et les héros vivaient encore parmi les hommes. Ces cités prétendent ainsi avoir été fondées par un dieu ou un héros mythique de cette époque. C'est ce qu'il est convenu d'appeler le passé mythique ou légendaire. Ces deux sortes de passé - historique d'un côté, mythique ou légendaire de l'autre - ne s'excluent pas mutuellement, mais coexistent aussi harmonieusement que possible. ${ }^{2}$ L'intérêt derrière la création d'un passé mythique est une question de prestige et de gloire civique. De cette façon, il est possible pour toute cité, même celle qui ne possède qu'un passé historique «banal», de se constituer un passé plus glorieux, mythique ou

\footnotetext{
* Il m'est agréable de montrer par cet article ma reconnaissance à Margarethe Billerbeck qui m'a connu jeune étudiant et qui m'a appris que la connaissance de l'Antiquité était indissociable de la rigueur philologique.

1 P. ex. pour Syracuse, cf. Bérard ( $\left.{ }^{2} 1957\right)$ 116-124, et pour Crotone, 151-157.

2 Ce phénomène de deux traditions contradictoires existant sans heurt apparent dans l'esprit humain n'est pas si inexplicable que ça. Que l'on songe que, de nos jours, mutatis mutandis, dans l'esprit de la plupart des gens coexistent, sans réel problème, la théorie de l'évolution et le récit biblique de la Création qui, pourtant, devraient s'exclure l'un l'autre. La plupart des gens ne se posent pas la question car ces deux théories appartiennent à des registres d'explication différents.
} 
légendaire. Ce phénomène est propre à la mentalité grecque. ${ }^{3}$ Cependant, durant l'époque hellénistique, qui possède un goût spécial pour les généalogies et les recherches érudites, il connaît un essor particulier et est établi selon des règles rigoureuses. Il se poursuit ainsi durant l'époque romaine. Par exemple, la cité de Tarse, dans la lointaine Cilicie, dont l'origine historique est à rechercher parmi les peuples du Moyen-Orient - puisqu'elle tire vraisemblablement son nom de leur dieu Tarku - prétendait avoir été fondée à l'époque mythique par le héros grec Persée, dont le pied (en

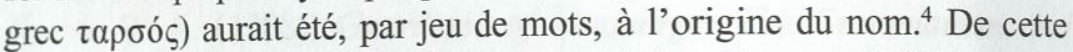
façon, bien que sise loin du cœur de l'Hellade, elle pouvait faire reconnaître son caractère grec..$^{5}$ Elle obtenait ainsi un certain lustre, renforcé par l'ancienneté qu'elle acquérait. Il convient de souligner que le rattachement à une cité grecque au moyen de la mythologie nécessitait beaucoup d'érudition. Tout ce travail sur la mythologie était pris très au sérieux par les Anciens. Bien que, de nos jours, ces mythes soient analysés avec un regard froid et que leur caractère légendaire soit évident, il n'est pas question pour autant de les mépriser. ${ }^{6}$ En analysant concrètement les liens de parenté que les mythographes antiques se sont ingéniés à trouver entre les héros mythiques des cités, on peut comprendre les rapports des héros entre eux et, par conséquent, ceux qu'entretenaient entre elles les cités dont les héros étaient apparentés.

3 Cf. Hdt. II,91, avec, pour la cité de Chemmis (Égypte), une version du mythe de Persée et de sa sandale que l'on retrouve pour la cité de Tarse (qui invoque, elle, le pied du héros). À ce sujet, voir ci-dessous, n. 4 et 5 .

4 Nonn. D. XVIII,291-294. Cf. l'étude de Robert (1977) 96-116, dont 108, n. 95.

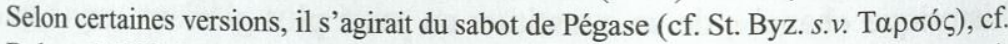
Robert (1977) 112-113, pour qui la légende de Pégase (et de Bellérophon) se serait imbriquée à celle de Persée.

5 Si elle se rattachait à Persée et, par son intermédiaire, à Argos, dont le héros était originaire, ce n'était pas par hasard, ni par pur caprice, car la région était particulièrement liée à Argos et revendiquait son lien de "parenté» avec cette cité grecque, cf. Robert (1977) 116-119, Stroud (1984; SEG 34,282), Curty (1995) 7-9, 13-15.

6 Même s'il est impossible de connaître l'opinion des Anciens sur ces récits légendaires, on sait que la plupart des gens y croyaient fermement comme on le voit par le nombre et la longévité de ces mythes. Nous sommes loin des remarques un brin méprisantes de Holleaux (1921) 54-55, n. 5, pour qui, à propos des parentés mythiques, les parties en présence «n'y regarderont pas de si près». Il faut plutôt adopter le point de vue de J. et L. Robert qui écrivaient (1983) 162, n. 3: «il ne faut pas les [i.e. les liens de parenté mythiques] traiter dans un esprit de frivolité dogmatique». 
Cependant, ce phénomène se transforma au fil du temps. Les cités, en effet, ne se contentèrent plus de ce que leur héros légendaire fût apparenté à celui d'une cité grecque quelconque; encore fallait-il que ce héros grec appartînt à une lignée prestigieuse et se rattachât à une cité célèbre comme Athènes, Sparte, Thèbes, etc.

Si l'on en revient à l'exemple de la Cilicie, on constate que l'histoire de Tarse, qui prétendait avoir été fondée par Persée, n'est pas un unicum dans la région. Il semble bien que des relations spécialement étroites aient relié la Cilicie et l'Argolide. ${ }^{7}$ Ainsi, la petite cité d'Aigée, en Cilicie, prétendait-elle aussi descendre de Persée. Ce héros était originaire d'Argos, cité fort prestigieuse de la Grèce. Par ce moyen, Aigée avait l'occasion de se hisser non seulement au niveau des cités grecques, mais de celles qui, parmi ces dernières, étaient les plus réputées et qui revendiquaient dans leur passé légendaire ce héros prestigieux. Ce phénomène, bien connu, a déjà fait l'objet d'un grand nombre de publications. ${ }^{8}$ C'est l'époque où il est, certes, prestigieux pour les cités de l'Empire romain de tirer leur origine d'une cité grecque, mais plus encore si celle-ci est célèbre. Cet aspect est connu sous le nom des «belles» parentés.

Le but de cet article est de montrer que les cités de l'île de Lesbos obéissent à cette même règle. C'est ainsi que, à côté de leur passé historique que je laisserai de côté, ${ }^{9}$ les quatre cités de l'île dont les noms sont Mytilène, Méthymna, Antissa et Érésos, cherchent, elles aussi, à se créer un passé légendaire. Par conséquent, elles revendiquent comme fondateur de chacune d'entre elles les quatre héros mythiques éponymes: la cité de Méthymna clame avoir été fondée par l'héroïne civique éponyme, Méthymne, tandis que les cités de Mytilène et Antissa font de même avec leur héroïne spécifique. Quant à la cité d'Érésos, étant donné son nom, elle ne peut revendiquer qu'un héros éponyme de sexe masculin.

Dans un second temps, après que ces traditions ont été bien établies, les cités tentent de donner du lustre au héros qui les a fondées. C'est pourquoi, les mythographes et autres historiens locaux essayèrent d'apparenter les quatre héros éponymes des cités à d'autres, plus prestigieux. De cette façon, ils cherchaient à ancrer les légendes de fondation de leur cité dans

Cf. ci-dessus, n. 5 .

8 Pour un status quaestionis, cf. Sartre (1991) 210. Cf. p. ex. Jones (1996) et Romeo (2002).

9 Pour de plus amples renseignements sur elles, cf. Labarre (1996) 14-19. 
un passé glorieux et à conférer à leur cité ainsi rehaussée, une renommée certaine et une autorité reconnue. De tous ces travaux, on trouve des traces dans les légendes de fondation de certaines cités chez différents auteurs, essentiellement des historiens grecs et latins ou chez des écrivains comme Strabon, Plutarque ou Pausanias. Mais c'est surtout Étienne de Byzance qui, en expliquant les différents ethniques, rapporte une foule de légendes de fondation et qui, sous cet aspect, constitue une mine de renseignements irremplaçable.

Si l'on en revient à Lesbos, parmi les héros prestigieux de l'île auxquels rattacher les héroïnes Méthymna, Mytilène, Antissa et le héros Érésos, se trouve la figure du roi Macar ou Macarée. ${ }^{10}$ L'autorité de ce roi mythique est incontestée car, preuve de son importance, son nom figure déjà dans l'Iliade ${ }^{11}$ ainsi que dans l'Hymne homérique à Apollon. ${ }^{12}$ Les mythographes utilisent donc le principe exposé à l'instant: ils s'emploient à rattacher les héros à Macar pour rehausser le lien familial de ces derniers et, de cette façon, rendre prestigieuse l'origine de la cité. Pour les héros Antissa et Érésos, bien que la tradition sur leur généalogie soit brève, c'est ainsi que les mythographes semblent agir. Dans une version transmise par une scholie à l'Iliade, ${ }^{13}$ Antissa passe pour être la femme de Macar et, dans une autre version dont Étienne de Byzance se fait l'écho, Érésos passe pour en être le fils. ${ }^{14}$ On peut tenir pour assuré que la version, faisant de l'héroïne Antissa la femme de Macar, provient de la cité d'Antissa tandis que celle où Érésos est le fils de Macar tire son origine de la cité d'Érésos. Une autre version, toujours transmise par la même scholie à l'Iliade, attribue cette fois comme mère à Macar l'héroöne Mytilène. Là aussi, nul doute que cette tradition ne provienne de la cité éponyme qui rehausse de cette façon sa propre origine. On le voit, le héros Macar est invoqué dans des généalogies différentes et apparaît chaque fois dans des

10 Il arrive assez fréquemment que Macar se voie confondu avec un autre héros appelé Macarée. C'est sans nul doute la quasi-homonymie entre les deux personnages qui a entraîné la confusion. Bien que les deux héros appartiennent à des généalogies fort distinctes, ils sont souvent donnés l'un et l'autre comme rois de l'île de Lesbos.

11 Hom. Il. XXIV,544.

12 h. Ap. 37.

13 Sch. ad Il. XXIV,544 (V 610 Erbse).

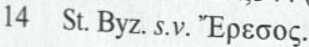


rôles autres, ce qui confirme l'observation de P. Mazon, l'éditeur d'Homère dans la $C U F .^{15}$

À côté de Macar, un deuxième héros mythologique, fort différent du premier, mais autant lié à l'île, est à prendre en considération: c'est Lesbos qui, comme le montre son nom, est le héros éponyme de l'île. Ce personnage possède aussi une envergure et une célébrité qui dépassent le cadre mythologique civique et local dans lequel sont confinés Méthymna, Mytilène, Antissa et Érésos, puisqu'il est le héros éponyme de l'île entière. C'est pourquoi, là aussi, comme pour Macar, les cités cherchent à rattacher, d'une manière quelconque, leur héros éponyme civique à Lesbos. Ainsi, par exemple, dans une tradition rapportée par Diodore de Sicile, ${ }^{16}$ l'héroïne civique Méthymna est-elle dite avoir épousé Lesbos. Il est clair qu'une telle légende provient de la cité de Méthymna. Là encore, en faisant de son éponyme la femme d'un des deux personnages mythiques les plus connus de l'île, la cité de Méthymna donne une renommée fort large à son héroïne et, du même coup, à elle-même.

C'est le même phénomène qui se produit en ce qui concerne cette fois la cité de Mytilène et son éponyme. On se rappelle la version déjà mentionnée dans laquelle Mytilène passait pour être la mère de Macar. Cependant, il y a d'autres versions qui rattachent l'héroïne Mytilène à Lesbos, cette fois. Comme Mytilène est la cité la plus grande et la plus connue de l'île, il semble que les mythographes se soient ingéniés à trouver diverses façons de rattacher son héroïne éponyme à des personnages bien connus de la mythologie et que, par conséquent, on ait des traces de plusieurs généalogies

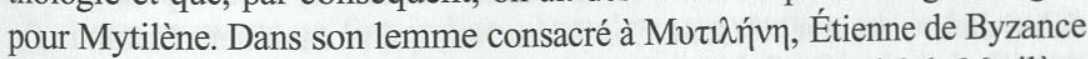
rapporte différentes versions. Il écrit que, selon les uns, la cité de Mytilène tirait son nom de son héroïne éponyme, fille de Macar ou de Pélops; que, selon d'autres, elle le tirait de son œciste ${ }^{17}$ Mytilès; que, selon d'autres enfin, c'était du fils de Poséidon et de Mytilène, Mytôn, qu'elle le tirait. Avec ces variantes, on constate que l'héroïne Mytilène, souvent à l'origine du nom de la cité, possède plusieurs généalogies dont certaines, prestigieuses, la placent au cœur de relations familiales illustres. Par ces travaux d'élaboration de

15 P. Mazon (1938) 159, n. 1: «Mais les diverses traditions relatives à la généalogie de ce personnage légendaire [= Macar] sont des plus contradictoires».

16 D. S. V,81,3-6.

17 J'emploie à dessein le terme «œciste» pour désigner de cette façon son fondateur «historique», différent de son fondateur «mythique». 
traditions prestigieuses, la cité de Mytilène ne ménage pas ses efforts pour élever socialement son héroïne éponyme et elle-même, par conséquent.

À l'issue de ce tour d'horizon, on est frappé par la richesse des mythes rattachant les éponymes locaux aux «grands» héros de l'île. Ces récits durent jouir d'une certaine popularité puisqu'on les retrouve jusque chez les auteurs antiques et leurs scholiastes. On constate autre chose: en tentant de rattacher plus ou moins adroitement son héros (ou héroïne) éponyme civique aux deux héros de l'île les plus célèbres, Macar et Lesbos, chacune des cités utilise des traditions mythiques qui divergent, voire qui s'opposent entre elles. Chaque cité, en effet, cherche à s'arroger, pour elle seule, les figures de Macar ou de Lesbos.

Cependant, à côté de ces récits contradictoires, les sources nous montrent aussi une tradition généalogique «égalitariste». ${ }^{18}$ Elle consiste à placer sur un pied d'égalité les quatre cités de l'île: elle fait des trois héroïnes et du héros éponymes les quatre enfants de Macar. Elle n'identifie plus une des héroïnes à la femme de ce dernier ou à celle de Lesbos ou une autre à sa mère. De cette manière, on ne remarque plus qu'un héros civique s'impose de façon plus prestigieuse par rapport aux trois autres. Au contraire, les quatre héros éponymes ont une seule tradition mythologique qui les décrit comme égaux. Pourquoi a-t-on introduit un tel système mythologique égalitariste? On admet que la mythologie a souvent pour but de justifier et d'expliciter la situation politique qui lui est contemporaine: l'apparition de cette tradition égalitariste s'expliquerait parfaitement comme étant le mythe officiel de la Confédération lesbienne, fondée peut-être au début du II $\mathrm{s}$. av. J.-C. ${ }^{19}$ Cette Confédération, qui se maintient durant la fin de l'époque hellénistique et jusque sous l'Empire romain, ${ }^{20}$ regroupe les

18 On peut voir une ébauche de ce mythe «égalitariste» chez Diodore de Sicile $(\mathrm{V}, 81,7)$ quand il affirme que Macar-Macarée eut d'autres filles que Mytilène et Méthymna. La formulation dans l'énoncé du mythe (qui provient certainement de la source de Diodore) est assez floue pour permettre aux cités d'Antissa et d'Érésos de rattacher leurs héros à Macar-Macarée. Cf. H. W. Stoll, s.v. Antissa, dans: Roscher (1884) I 1,385 et A. Schultz, s.v. Eresos, dans: Roscher (1884) I 1,1300.

19 La date de sa fondation est controversée. Il peut s'agir soit de la fin du $\mathrm{III}^{\mathrm{e}} \mathrm{s}$ s. av. J.-C., soit du début du II e, en tout cas avant 167 av. J.-C. (voir ci-dessous). Le premier document à attester l'existence de la Confédération est $I G$ XII Suppl., 136, repris par IG XI 4,1064, cf. Labarre (1994) 426-427.

20 Là non plus, aucune date précise ne peut être avancée. On supposait auparavant la disparition de la Confédération à la fin du III//début du $\mathrm{IV}^{\mathrm{e}} \mathrm{s}$. apr. J.-C., mais selon 
cités de l'île dans un système politique où chacune a une importance égale aux autres. Son existence a été mise en lumière par L. Rober ${ }^{21}$ et son fonctionnement étudié par G. Labarre, il y a presque vingt ans. ${ }^{22}$ On comprend aisément qu'une telle confédération, qui rend égales entre elles les cités de l'île, ait recours à un système mythologique faisant des héroïnes et du héros éponymes des cités non seulement des sœurs et frère, mais aussi des enfants de Macar, le roi de l'île, dans le but d'assurer sa propre légitimité.

Cependant, cette généalogie égalitariste a dû recourir à un subterfuge pour s'imposer: si on constate que le statut de Macar reste identique à celui qu'il a toujours été et qu'atteste l'Iliade, celui de Lesbos, en revanche, se modifie. Les mythographes ont pu facilement changer celui de Lesbos car, à la différence de Macar, il n'a pas un statut clair, entériné par la tradition écrite. Par conséquent, ils l'ont transformé en en faisant une femme qui devient l'épouse de Macar. Il ou elle, ainsi transformé(e), reste bien sûr l'éponyme de l'île, mais ce n'est qu'au prix de sa masculinité qu'il ou elle peut être placé(e) au même rang que Macar.

La tradition faisant des quatre héros éponymes des cités de l'île les enfants de Macar et Lesbos peut être datée. En effet, elle mentionne l'existence d'Antissa, dont la destruction et la suppression par les Romains date de 167 av. J.-C., ${ }^{23}$ ce qui constitue forcément le terminus ante quem du mythe. Ce fait concorde avec la création de la Confédération elle-même qui eut lieu peu auparavant. ${ }^{24}$ Nous relions ces deux faits et affirmons que la mythologie, faisant de Macar le père des quatre éponymes des cités, est celle qui était officiellement reconnue par la Confédération lesbienne.

Nous en avons un fort indice, si ce n'est une preuve, par le fait que la Confédération utilisait la version mythologique où Lesbos était la femme de Macar. En effet, elle n'hésite pas à donner à un couple de bienfaiteurs du II $\mathrm{e}$ s. apr. J.-C. le titre de «nouveau Macar» et de «nouvelle Lesbos». ${ }^{25}$ C'est ainsi que, grâce aux attestations ténues que l'on trouve dispersées dans diverses sources, j'ai pu mettre en évidence la mythologie utilisée par la

Labarre (1994) 445-446, il n'y a pas d'argument en faveur de sa disparition à cette époque.

21 Robert (1925).

22 Labarre (1994) 426-446.

23 Liv. XLV,31,14.

24 Voir ci-dessus, n. 19.

25 Robert (1960) 309-311. 
Confédération lesbienne. Celle-ci, bien connue institutionnellement depuis les travaux de L. Robert et ceux, plus récents, de G. Labarre, se voit attribuer une mythologie qu'elle possédait déjà mais qui, cachée dans diverses sources, n'apparaissait pas clairement. Elle semble maintenant être assurée.

\section{Bibliographie}

Bérard, J. ( ${ }^{1957), ~ L a ~ c o l o n i s a t i o n ~ g r e c q u e ~ d e ~ l ' I t a l i e ~ m e ́ r i d i o n a l e ~ e t ~ d e ~ l a ~}$ Sicile dans l'Antiquité. L'histoire et la légende, Paris.

Curty, O. (1995), Les parentés légendaires entre cités grecques. Catalogue

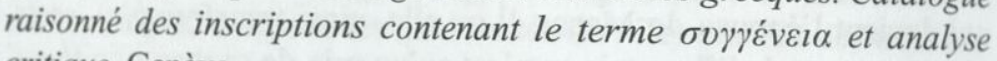
critique, Genève.

Holleaux, M. (1921), Rome, la Grèce et les monarchies hellénistiques, Paris.

Jones, C. P. (1996), «The Panhellenion», Chiron 26, 29-56.

Labarre, G. (1994), «KOINON $\Lambda \mathrm{E} \Sigma \mathrm{BI} \Omega \mathrm{N} », R E A$ 96, 415-446.

Labarre, G. (1996), Les cités de Lesbos aux époques hellénistique et impériale, Lyon.

Mazon, P. (1938), Homère, Iliade, texte établi et traduit, t. IV, Paris.

Robert, J. et L. (1983), Fouilles d'Amyzon en Carie, t. I, Paris.

Robert, L. (1925), «Lesbiaca», REG 38, 29-43 (= Opera minora selecta, Amsterdam 1969, t. II, 721-735).

Robert, L. (1960), «V. Inscriptions de Lesbos», REA 62, 285-315 (=Opera minora selecta, Amsterdam 1969, t. II, 801-831).

Robert, L. (1977), «IV. Deux inscriptions de Tarse et d'Argos», BCH 101, 88-132 (= Documents d'Asie Mineure, Paris 1987, 46-90).

Romeo, I. (2002), «The Panhellenion and ethnic identity in Hadrianic Greece», CPh 97, 21-40.

Roscher, W. H. (éd.) (1884-1937), Ausführliches Lexikon der griechischen und römischen Mythologie, 7 vols., Leipzig.

Sartre, M. (1991), L'Orient romain: Provinces et sociétés provinciales en Méditerranée orientale, Paris.

Stroud, R. S. (1984), «An Argive decree from Nemea concerning Aspendos», Hesperia 53, 191-216. 